\title{
Report of the Skin Research Workgroups from the GRAPPA 2019 Annual Meeting
}

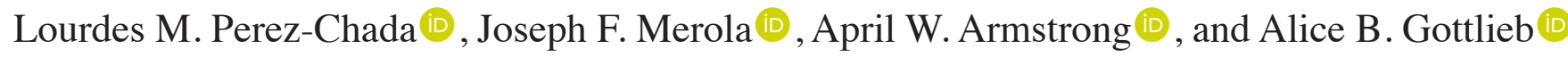

\begin{abstract}
The International Dermatology Outcome Measures (IDEOM) initiative is a nonprofit organization dedicated to enhancing clinical care and research in dermatology by developing evidence-based, patient-centered outcome measures. At the 2019 annual meeting of the Group for Research and Assessment of Psoriasis and Psoriatic Arthritis (GRAPPA), the IDEOM psoriasis working group presented an overview of its selected deliverables and discussed its efforts to agree on meaningful, valid, and feasible outcome measures for quality measurement in psoriasis. The psoriatic arthritis (PsA) workgroup focused on the measurement of PsA symptoms in psoriasis clinical trials, and the measurement of nonspecific musculoskeletal symptoms among patients with psoriasis in psoriasis longitudinal clinical trials and cohort studies. (J Rheumatol Suppl. 2020 June;96:36-40; doi:10.3899/ jrheum.200125)
\end{abstract}

Key Indexing Terms: PSORIATIC ARTHRITIS PSORIASIS QUALITY MEASURE

GRAPPA IDEOM MUSCULOSKELETAL

\section{The International Dermatology Outcome Measures (IDEOM) Initiative: Mission and Evolution}

The IDEOM initiative is a nonprofit organization that was founded in 2013 with the mission to establish patient-centered measurements to enhance research and treatment for those with dermatologic disease ${ }^{1}$. Toward this goal, IDEOM has established multiple workgroups and collaborations (Table 1).

The psoriasis workgroup was the first workgroup to be established, with support from the National Psoriasis Foundation $(\mathrm{NPF})^{2}$. After 4 years of continued work involving literature review, live meetings with nominal group discussions, pre-Delphi electronic surveys, conference calls, and

From the Department of Dermatology and Medicine, Division of Rheumatology, Harvard Medical School, Brigham and Women's Hospital, Boston, Massachusetts; Department of Dermatology, Icahn School of Medicine at Mt. Sinai, New York, New York; Department of Dermatology, Keck School of Medicine, University of Southern California (USC), Los Angeles, California, USA.

As part of the supplement series GRAPPA 2019, this report was reviewed internally and approved by the Guest Editors for integrity, accuracy, and consistency with scientific and ethical standards.

Dr. Armstrong has served as a research investigator or consultant to Leo, AbbVie, UCB, Janssen, Lilly, Novartis, Ortho Dermatologics, Sun, Dermavant, BMS, Sanofi, Regeneron, Dermira, and Modmed.

L.M. Perez-Chada, MD, Department of Dermatology, Harvard Medical School, Brigham and Women's Hospital; J.F. Merola, MD, Department of Dermatology and Medicine, Division of Rheumatology, Harvard Medical School, Brigham and Women's Hospital; A.W. Armstrong, MD, MPH, Department of Dermatology, Keck School of Medicine, USC; A.B. Gottlieb, MD, PhD, Department of Dermatology, Icahn School of Medicine at Mt. Sinai.

Address correspondence to Dr. A.B. Gottlieb, MD, PhD, Clinical Professor of Dermatology, Icahn School of Medicine at Mt. Sinai, Department of Dermatology, 10 Union Square East, New York, New York 10003,USA.E-mail: alice.gottlieb@mountsinai.org a 2-round electronic Delphi survey, the workgroup established a Core Domain Set for psoriasis clinical trials ${ }^{3}$. This Core Domain Set includes the domains of skin manifestations, investigator and patient's global assessment (PtGA), psoriasis and psoriatic arthritis (PsA) symptoms, treatment satisfaction, and health-related quality of life (QOL). New workgroups were then formed to identify appropriate outcome measures for each specific domain.

Next, IDEOM allied with the Acne Core Outcomes Research Network to establish the acne workgroup. In addition, IDEOM partnered with the Cochrane Skin GroupCore Outcome Set Initiative and the Zealand University Hospital (Roskilde, Denmark) to found the Hidradenitis Suppurativa Core Outcomes Set International Collaboration (HISTORIC) ${ }^{4}$. Mirroring the psoriasis workgroup, HISTORIC has also defined a Core Domain Set for hidradenitis suppurativa clinical trials that includes the domains of physical signs, hidradenitis suppurativa-specific QOL, global assessment, pain, progression of disease course, and symptoms of hidradenitis suppurativa ${ }^{5}$. Last, IDEOM allied with the American Academy of Dermatology (AAD) to advance outcome measurement in clinical practice ${ }^{6,7}$.

In this report, we present an overview of IDEOM's selected activities that were presented at the 2019 Group for Research and Assessment of Psoriasis and PsA (GRAPPA) annual meeting in Paris, France.

\section{IDEOM's PsA Symptoms Workgroup}

The IDEOM psoriasis workgroup has included PsA symptoms in the Core Domain Set to be measured in all psoriasis clinical trials ${ }^{3}$. The PsA symptoms workgroup was then established to define the measurement of PsA symptoms in this context. 
Table 1. IDEOM's workgroups and collaborations.

Psoriasis Workgroup

Psoriasis Core Domain Set

- Domains that are considered required to measure in every psoriasis

clinical trial:

1. Skin manifestations

2. Primary (BSA/erythema/induration/scale)

3. Location of skin lesions

a. Palmar-plantar psoriasis

b. Scalp psoriasis

4. Investigator global

5. Psoriasis and PsA symptoms

6. PtGA

7. Treatment satisfaction

8. HRQOL

- Domains that are not required but may be important depending on the study objectives:

1. Skin manifestations

a. Nail psoriasis

b. Inverse psoriasis

c. Genital psoriasis

d. Guttate psoriasis

e. Secondary manifestations

- Domains that are considered for the research agenda:

1. PsA signs

2. Economic effect

a. Direct cost

b. Indirect cost

3. Work productivity/participation

4. Cardiovascular disease

Workgroups established to identify the best-suited outcome measurement instruments for each core domain:

1. Treatment Satisfaction Workgroup: An SLR of the measurement properties of treatment satisfaction instruments used in dermatology revealed that neither of the existing instruments meet the minimum quality criteria standards. A new treatment satisfaction instrument is under development.

2. PsA Symptoms Workgroup: Through a Delphi consensus exercise, it was agreed that all patients enrolling in a psoriasis clinical trial should be screened for PsA and that the PsAID-9 meets the necessary quality criteria standards to measure PsA symptoms in psoriasis clinical trials. The RAPID-3 was considered an acceptable alternative pending further validation.

3. Investigator PtGA Workgroup: An SLR of the measurement properties of global assessment instruments did not identify instruments meeting the minimum quality criteria standards. A consensus exercise to define next steps is under way.

4. HRQOL Workgroup: An SLR of the measurement properties of HRQOL measures is under way.

Hidradenitis Suppurativa Workgroup (HISTORIC)

Hidradenitis Suppurativa Core Domain Set

- Domains that are considered required to measure in every psoriasis clinical trial:

1. Pain

2. Physical signs
a. Anatomic location
b. Surface area
c. Total lesion count
d. Inflammatory lesion count
e. Number of abscesses

Table 1. Continued.

f. Number of inflamed nodules

g. Number of sinus tracts

h. Number of fistulae

3. Hidradenitis Suppurativa QOL

a. Physical functioning

b. Psychological functioning

c. Psychosocial functioning

d. Emotional well-being

e. Ability to work or study

4. Global assessment

a. PtGA

b. Investigator global

5. Progression of course

a. Flare frequency and duration

b. Time to recurrence

-Domains that reached "consensus in" for patients or healthcare providers only:

1. Symptoms ${ }^{1}$

a. Drainage

b. Fatigue

2. Sleep disturbance

3. Number of chronic areas

- Domains that are considered for the research agenda or important in specific trials:

1. Biomarkers

2. Time to heal

Workgroups were established to identify the best-suited outcome measurement instruments for each core domain. When measures do not exist for the specified items, or when measures may not be sufficiently validated, workgroups have been charged with developing new measures.

1. Hidradenitis Suppurativa-Specific QOL Workgroup: HISTORIC has developed the HiSQOL, the first disease-specific QOL instrument in hidradenitis suppurativa. HiSQOL has been accepted into the FDA qualification program and will be further tested in phase III trials.

2. Investigator Global Assessment Workgroup: An Investigator Global Assessment for Hidradenitis Suppurativa was developed in PIONEER I study and will be validated in PIONEER II, as well as externally.

Acne Workgroup: ACORN

Acne Core Domain Set

Domains that are considered required to measure in every acne clinical trial:

1. Satisfaction with appearance

2. HRQOL

3. Extent of scars/dark marks

4. Signs and symptoms

5. Adverse events

6. Satisfaction with treatment received

7. Longterm control of acne

\section{IDEOM-AAD Collaboration}

IDEOM collaborated with the AAD to meet consensus on valid, feasible, and meaningful outcome measures that could be incorporated into quality measures for inflammatory dermatoses. The collaboration conducted 2 consecutive modified Delphi processes:

1. Delphi process to achieve consensus on a physician-assessed outcome measure.

a. Consensus was reached for a single, 5-point ordinal physician global assessment instrument without anchoring descriptors to measure clinical outcomes in acne, eczema, and psoriasis patients: 
clear $=0 ;$ almost clear $=1 ;$ mild $=2 ;$ moderate $=3 ;$ severe $=4$. b. Descriptors could potentially be provided to individual groups on a local level to guide disease severity assessment.

2. Delphi process to achieve consensus on a patient-reported outcome measure.

a. A combination of a PtGA with Skindex instruments (Skindex-16 or Skindex Mini) or other measure of HRQOL was recommended to measure clinical outcomes in acne, eczema, and psoriasis patients b. Future work will focus on conducting further evaluation of the measurement properties of identified outcome measures and incorporating them into quality measures.

NAAF Collaboration

Development of a patient-reported outcome measure for alopecia areata.

1 "Symptoms" is recommended as a Core Domain by the HISTORIC Steering Group because it is a patient-reported domain that received strong support from the patient group. IDEOM: International Dermatology Outcome Measures; BSA: body surface area; PsA: psoriatic arthritis; PtGA: patient's global assessment; QOL: quality of life; HRQOL: health-related QOL; SLR: systematic literature review; PsAID-9: Psoriatic Arthritis Impact of Disease 9; RAPID-3: Routine Assessment of Patient Index 3; HISTORIC: HIdradenitis SuppuraTiva cORe outcomes set International Collaboration; HiSQOL: Hidradenitis Suppurativa QOL instrument; FDA: US Food and Drug Administration; ACORN: Acne Core Outcomes Research Network; AAD: American Academy of Dermatology; NAAF: National Alopecia Areata Foundation.

In 2018, the workgroup conducted a Delphi consensus exercise in which 297 international participants agreed that (1) all psoriasis trial participants should be screened for PsA prior to the measurement of PsA symptoms; (2) the measurement of PsA symptoms should occur in those who screen positive or have a prior rheumatologist diagnosis of PsA; and (3) the most appropriate instrument to measure PsA symptoms in psoriasis clinical trials is the PsA Impact of Disease 9 (PsAID-9), with the Routine Assessment of Patient Index Data 3 representing an acceptable alternative ${ }^{8,9}$.

Following this exercise, the workgroup sought to define how to measure musculoskeletal (MSK) symptoms (e.g., pain, weakness, stiffness, fatigue) in psoriasis subjects who have not yet developed concomitant PsA. Because we know that patients with psoriasis experience nonspecific MSK symptoms and that about $30 \%$ of them will eventually develop PsA ${ }^{10}$, the identification and quantification of MSK symptoms/complexes of symptoms in patients with psoriasis holds great potential to predict and prevent the onset of PsA.

Longitudinal cohort studies and longterm clinical trials involving patients with psoriasis represent an ideal setting to measure MSK symptoms and to monitor the transition into PsA. These data can eventually be used to develop predictive algorithms for PsA. However, the measurement of MSK symptoms faces multiple barriers. First, consensus needs to be reached on which MSK symptoms should be measured. One prospective cohort study involving 410 patients with psoriasis, of which 57 developed PsA, iden- tified that the presence of arthralgia (in women), heel pain, fatigue, and stiffness were predictors of PsA ${ }^{11}$. However, further studies are warranted to confirm these findings. Second, patients will need to be classified at baseline as having PsA or not, but in the absence of a diagnostic test for PsA and the limited access to rheumatologists, classification relies on screening tools that have relatively low specificity ${ }^{12}$. As a consequence, screening tools could introduce selection bias because they may misclassify patients who have MSK symptoms due to other noninflammatory rheumatologic conditions such as fibromyalgia (FM) and osteoarthritis (OA). Third, validated instruments to measure MSK symptoms in psoriasis subjects need to be developed or identified. The validation of instruments that were originally developed to measure MSK symptoms in PsA could be considered, but these may need to be adjusted for patients with psoriasis. For example, the stem of the questions in the PsAID- $9^{13}$ refers to "known" PsA, which is not appropriate for patients with psoriasis (e.g., circle the number that best describes the pain you felt because of your PsA).

At the 2019 IDEOM Annual Meeting in Washington, D.C., the PsA symptoms workgroup discussed the advantages and limitations of measuring MSK symptoms in psoriasis and asked participants to vote on a set of pre-established questions using an automated response system (ARS). In all, $80 \%$ agreed that there is a need to confirm the presence of other potential confounding MSK disease (e.g., FM and OA) when assessing PsA symptoms in patients with psoriasis. In addition, $60 \%$ voted that current clinical PsA screening tools are not specific enough for classifying patients in a psoriasis research study; $72 \%$ agreed that novel tools with high specificity, approaching that of a diagnostic test, need to be developed for research purposes. Finally, 62-63\% believed that it is feasible and valid to ask nonrheumatologists to measure signs of inflammatory arthritis (i.e., tender and swollen joint count, enthesitis).

The PsA symptoms workgroup is actively working to define the best approach to measure MSK symptoms in psoriasis clinical studies. The workgroup is conducting a critical review of existing MSK symptom measures. Additionally, it plans to conduct a qualitative study to determine which MSK symptoms are meaningful to patients with psoriasis and how these differ from those experienced by patients with established PsA. Results of these efforts will be presented at the 2020 IDEOM annual meeting in Washington, D.C.

\section{IDEOM-AAD Collaboration for Clinical Practice}

In the era of value-based medicine, dermatologists need to prove the value of the care they provide to their patients, policy makers, and payers ${ }^{14}$. Because value is defined as the outcomes achieved divided by the cost of the whole cycle of care for the patient's medical condition ${ }^{15}$, dermatologists need to implement the collection of standardized, mean-

Personal non-commercial use only. The Journal of Rheumatology Copyright $($ C 2020. All rights reserved. 
ingful clinical outcome measures to demonstrate value. In collaboration with the AAD, IDEOM conducted 2 consecutive modified Delphi exercises to reach consensus on valid, feasible, and meaningful outcome measures that could be incorporated into quality measures for chronic inflammatory dermatoses.

First Delphi exercise: global assessor severity measure. The first Delphi exercise occurred in February 2018 in New York and focused on reaching consensus on a provider-reported global disease severity measure. Through an anonymous ARS, collaborators voted on (1) which inflammatory dermatoses should be assessed by a global measure, and (2) which scale type would be more appropriate to quantify global disease severity (i.e., a numerical rating scale, a dichotomous clear/almost clear vs not outcome, or a 5-point Likert scale with or without descriptors). When evaluating different Likert scales for the voting exercise, meeting leaders favored a 5-point Likert scale (clear $=0$, almost clear $=1$, mild $=2$, moderate $=3$, severe $=4$ ) over a 6-point scale (clear $=0$, almost clear $=1$, mild $=2$, moderate $=3$, severe $=4$, very severe $=5$ ) because the extreme option "very severe" is rarely used by clinicians in clinical practice, and most clinical trials have used the 5-point scale. For the first question, consensus was achieved when $>90 \%$ of participants agreed on an inflammatory dermatosis. For the second question, consensus was achieved when $>70 \%$ of collaborators agreed on a scale type.

In all, 1 patient, 1 research fellow, 18 dermatologists, 6 rheumatologist-dermatologists, 3 pediatric dermatologists, and 7 quality measures experts attended the meeting. Psoriasis, atopic dermatitis, and acne were selected among 23 inflammatory skin diseases discussed as the inflammatory dermatoses that should be assessed by a global measure. Collaborators agreed on a 5-point ordinal scale for quantification methods (clear $=0$, almost clear $=1$, mild $=2$, moderate $=3$, severe $=4$ ) without anchoring language. However, consensus was also reached to develop and validate disease-specific anchoring descriptors to guide clinicians in their assessment of disease severity. These descriptors could eventually be provided through referenced electronic platforms (e.g., AAD Website, Internet applications) ${ }^{16}$.

At the 2018 IDEOM annual meeting in Washington, D.C., IDEOM presented the Delphi results to 86 international participants who were then queried about the implementation of physician-assessed and patient-reported outcome measures (PROM) in clinical practice using ARS. In all, $93.5 \%$ and $88 \%$ of participants agreed that a simple physician-assessed and a patient-reported outcome measure for treatment response, respectively, should be incorporated into every patient medical record. In addition, $81.6 \%$ voted that these should not be combined into a single composite score. It was further agreed that payers should not base decisions to cover treatment costs solely upon the score of a patient-reported instrument $(96 \%)$, and that payers should not determine reimbursements based solely on physician-assessed outcome measures (86\%). Finally, collaborators agreed that the goal of treat-to-target strategies should be to maximize both physician-assessed and PROM (71\%).

Second Delphi Exercise: PROM. The second Delphi exercise aimed to achieve consensus on a PROM for clinical practice. Participants including patients with acne, psoriasis, and atopic dermatitis; dermatologists; a nondermatologist physician; patient association representatives; and members of the AAD $(n=53)$ met in October 2018 in Chicago, Illinois, and discussed advantages and disadvantages of Skindex instruments ${ }^{17}$ and PtGA for inflammatory skin conditions. Participants then voted on different aspects of these measures using a modified ARS. Consensus was defined as $\geq 70 \%$ of agreement within the group.

Skindex instruments are patient-reported instruments that allow for a comprehensive assessment of a patient's disease burden by evaluating 3 distinct domains: symptoms, emotions, and functioning ${ }^{17}$. There are 3 Skindex versions available, including the Skindex-29, Skindex-16, and Skindex-mini (3 questions) $)^{18}$. At this meeting, collaborators agreed that patients should ideally complete the Skindex-16 at their first visit to the dermatologist and then the Skindex-mini in followup visits $(75 \%)$. When compared to PtGA, $74 \%$ of participants preferred Skindex instruments.

In evaluating PtGA, $84 \%$ of participants endorsed the following candidate instrument: "Overall, how severe is your skin disease today?"; response options: " $0=$ clear, 1 = almost clear, 2 = mild, 3 = moderate, $4=$ severe," with an optional check-box: "worst ever." This optional checkbox was adopted from the NPF Psoriasis Scale. In addition, $84.4 \%$ of participants favored the inclusion of an instrument that could determine change over time: "Since starting treatment, my skin disease is:"; response options: "the same," "improving," and "worsening."

Finally, collaborators were queried about the role of PROM in clinical practice, with $72 \%$ voting that they are important to initiate conversations about the effect of disease between the patient and the clinician. Additionally, 74\% agreed that the quality of a physician should not depend on the score of PROM, given that this score could be influenced by several other factors such as the severity of the underlying disease, social/family support, access to therapies, and comorbidities.

Therefore, the IDEOM-AAD resolved that PtGA should be complemented with the Skindex instrument or other multidimensional measure of health-related QOL to identify domains of health that are relevant to patients.

\section{DISCUSSION}

In the absence of standardized, patient-centered outcome measures in psoriasis, IDEOM is actively working to define meaningful outcome measures for both clinical trials and

Personal non-commercial use only. The Journal of Rheumatology Copyright @ 2020 . All rights reserved. 
clinical practice. This report provides an update on IDEOM's work presented at the GRAPPA 2019 annual meeting. For psoriasis clinical trials, IDEOM has defined a Core Domain Set and is now working to identify the most appropriate outcome measures for each domain. The PsAID-9 was selected as the most appropriate instrument for the measurement of the PsA symptoms domain. Ongoing efforts are centered on identifying a meaningful, patient-centered measure for MSK symptoms in patients with psoriasis. For psoriasis clinical practice, the IDEOM-AAD alliance agreed on a 5-point ordinal physician's global assessment instrument and a combination of a PtGA with Skindex instruments or other measures of health-related QOL to measure clinical outcomes. Future work will focus on conducting further validation of these outcome measures and incorporating them into quality measures.

\section{REFERENCES}

1. Gottlieb AB, Levin AA, Armstrong AW, Abernethy A, Duffin $\mathrm{KC}$, Bhushan R, et al. The International Dermatology Outcome Measures Group: formation of patient-centered outcome measures in dermatology. J Am Acad Dermatol 2015;72:345-8.

2. Gottlieb AB, Armstrong AW, Christensen R, Garg A, Duffin $\mathrm{KC}$, Boehncke WH, et al. The International Dermatology Outcome Measures initiative as applied to psoriatic disease outcomes: a report from the GRAPPA 2013 meeting. J Rheumatol 2014;41:1227-9.

3. Callis Duffin K, Merola JF, Christensen R, Latella J, Garg A, Gottlieb AB, et al. Identifying a Core Domain Set to assess psoriasis in clinical trials. JAMA Dermatol 2018;154:1137-44.

4. Thorlacius L, Ingram JR, Garg A, Villumsen B, Esmann S, Kirby JS, et al. Protocol for the development of a core domain set for hidradenitis suppurativa trial outcomes. BMJ Open 2017;7:e014733.

5. Thorlacius L, Ingram JR, Villumsen B, Esmann S, Kirby JS, Gottlieb AB, et al; HIdradenitis SuppuraTiva cORe outcomes set International Collaboration (HISTORIC). A core domain set for hidradenitis suppurativa trial outcomes: an international Delphi process. Br J Dermatol 2018;179:642-50.

6. Gottlieb A, Salame N, Armstrong AW, Merola JF, Parra S, Takeshita $\mathrm{J}$, et al; International Dermatology Outcome Measures and the American Academy of Dermatology. A provider global assessment quality measure for clinical practice for inflammatory skin disorders. J Am Acad Dermatol 2019;80:823-8.
7. Perez-Chada L, Taliercio VL, Gottlieb A, Van Beek M, Duffin KC, Reeder M, et al. Achieving consensus on patient-reported outcome measures in clinical practice for inflammatory skin disorders: on behalf of International Dermatology Outcome Measures and the American Academy of Dermatology. J Am Acad Dermatol 2019 Sep 13 (E-pub ahead of print).

8. Perez-Chada LM, Cohen JM, Gottlieb AB, Duffin KC, Garg A, Latella $\mathrm{J}$, et al. Achieving international consensus on the assessment of psoriatic arthritis in psoriasis clinical trials: an International Dermatology Outcome Measures (IDEOM) initiative. Arch Dermatol Res 2018;310:701-10.

9. Perez-Chada LM, Gottlieb AB, Cohen J, Mease P, Duffin KC, Garg A, et al. Measuring psoriatic arthritis symptoms: a core domain in psoriasis clinical trials. J Am Acad Dermatol 2020;82:54-61.

10. Karreman MC, Weel AE, van der Ven M, Vis M, Tchetverikov I, Nijsten TE, et al. Prevalence of psoriatic arthritis in primary care patients with psoriasis. Arthritis Rheumatol 2016;68:924-31.

11. Eder L, Polachek A, Rosen CF, Chandran V, Cook R, Gladman DD. The development of psoriatic arthritis in patients with psoriasis is preceded by a period of nonspecific musculoskeletal symptoms: a prospective cohort study. Arthritis Rheumatol 2017;69:622-9.

12. Ogdie A, Weiss P. The epidemiology of psoriatic arthritis. Rheum Dis Clin North Am 2015;41:545-68.

13. Gossec L, de Wit M, Kiltz U, Braun J, Kalyoncu U, Scrivo R, et al; EULAR PsAID Taskforce. A patient-derived and patient-reported outcome measure for assessing psoriatic arthritis: elaboration and preliminary validation of the Psoriatic Arthritis Impact of Disease (PsAID) questionnaire, a 13-country EULAR initiative. Ann Rheum Dis 2014;73:1012-9.

14. Resneck JS Jr., VanBeek M. What dermatology still needs to create meaningful patient outcome measurements. JAMA Dermatol 2015;151:371-2.

15. Porter ME. What is value in health care? N Engl J Med 2010;363:2477-81.

16. Gottlieb A, Salame N, Armstrong AW, Merola JF, Parra S, Takeshita $\mathrm{J}$, et al; International Dermatology Outcome Measures and the American Academy of Dermatology. A provider global assessment quality measure for clinical practice for inflammatory skin disorders. J Am Acad Dermatol 2019;80:823-8.

17. Chren MM. The Skindex instruments to measure the effects of skin disease on quality of life. Dermatol Clin 2012;30:231-6.

18. Swerlick RA, Zhang C, Patel A, Chren MM, Chen S. The Skindex-mini: a streamlined QOL measurement tool suitable for routine use in clinic. J Am Acad Dermatol 2018 Dec 24 (E-pub ahead of print). 